\title{
189. Solvent Perturbation of Tryptophan Residue in Polyamino Acid
}

\author{
By Katsuya Hayashi, *) Takachika AzUma, **) Akio OHnishi, **) \\ and Junzo NoGUCHI**)
}

(Comm. by Yuzuru OkUdA, M. J. A., Oct. 12, 1968)

Polymer catalyzers including the enzyme have been characterized by the participation of their polyfunctional sites in the catalytic activity. The formation of the polyfunctional site may depend upon the structure and flexibility of the main chain of polymers. Although many studies on the secondary structure of the polyamino acid have been carried out, ${ }^{1)}$ the relation between the structure of polypeptide chain and the formation of polyfunctional site has not been established. In the present study, the solvent-accessibility of the tryptophan residues of polyamino acids was estimated by solvent perturbation to accumulate informations about polyfunctional site formation.

The other purpose was the characterization of a peak around $230 \mathrm{~m} \mu$ in the difference spectrum of a polymer containing tryptophan residues. A change in optical density of a peak around $230 \mathrm{~m} \mu$ in the difference spectrum of protein has been believed to be index of the change in secondary structure of polypeptide chain. ${ }^{2)}$ However, the change in the optical density around $230 \mathrm{~m} \mu$ may also be caused by (1) the effect of the ionization state of adjacent groups on the tryptophan chromophore ${ }^{3)}$ and (2) the exposure of buried tryptophan residue to solvent. ${ }^{4}$ In order to characterize the peak around $230 \mathrm{~m} \mu$ in the difference spectrum, the correlation of the intensity around $230 \mathrm{~m} \mu$ to that at $293 \mathrm{~m} \mu$ was analyzed by the solvent perturbation technique.

Materials and methods. (1) Copolymer of tryptophan, alanine and glutamic acid. N-Carboxyanhydrides of L-tryptophan, L-alanine and $\gamma$-methyl-L-glutamate were synthesized by introducing phosgene gas in the amino acid suspensions in dioxane. ${ }^{1)}$ The $\mathrm{N}$-carboxyanhydrides in anhydrous dioxane were copolymerized at $40^{\circ} \mathrm{C}$ by addition of triethylene diamine. The copolymer was saponified by a slight excess of $0.2 \mathrm{~N}$ sodium hydroxide in $80 \%$ methanol at $15^{\circ} \mathrm{C}$. The resulted polymer was purified by acid-precipitation.

(2) Solvent perturbation. For the measurement of difference

\footnotetext{
*) Laboratory of Biochemistry, Faculty of Agriculture, Kyushu University, Fukuoka.

**) Department of Polymer Science, Faculty of Science, Hokkaido University, Sapporo.
} 
spectrum around $230 \mathrm{~m} \mu$, the UV-spectrum of tryptophan $\left(3.95 \times 10^{-4}\right.$ $\mathrm{M})$, the polymer $\left(3.75-3.95 \times 10^{-4} \mathrm{M}\right)^{*)}$ or lysozyme $\left(6.6 \times 10^{-5} \mathrm{M}\right)$ dissolved in the glucose or sucrose solution, was compared with that of an aqueous solution of tryptophan, the polymer or lysozyme at the same concentration. For the difference spectrum around $230 \mathrm{~m} \mu, 3.5$ times diluted solution was used. The degree of solvent-accessibility of tryptophan residue was calculated according to the method described in a previous paper. ${ }^{5)}$

(3) Charge effect. UV-spectra of $1.18 \times 10^{-4} \mathrm{M}$ polymer solution at pHs 5.0 and 6.0 were compared with that at $\mathrm{pH} 8.0$.

(4) Optical rotatory dispersion of the polymer solution (3.9-6.5 $\left.\times 10^{-3} \mathrm{M}\right)$ at $\mathrm{pH} 5.0$ was measured in the range of wave length from 300 to $600 \mathrm{~m} \mu$. The parameter $\mathrm{b}_{0}$ was calculated by Moffitt-Yang equation. ${ }^{6)}$

Results. Solvent perturbation of poly $\operatorname{Trp}^{5} A l a^{48} G l u^{48 * *)}$ (PI) by glucose. A typical difference spectrum of $3.95 \times 10^{-4} \mathrm{M}$ PI in solvent perturbation is shown in Fig. 1. The optical densities of peaks are plotted as a function of glucose concentration in Fig. 2. $\Delta \mathrm{OD}_{235}{ }^{\prime} \mathrm{s}^{* * *}$ ) of PI did not intercept the origin. This anomaly might be caused by an excessive optical density at $235 \mathrm{~m} \mu$, because, when a solution with optical density larger than 2.5 was applied for difference spectrum the machine generally could not respond regularly. It is therefore conceivable that several times dilution of solute will make a good match of the concentration

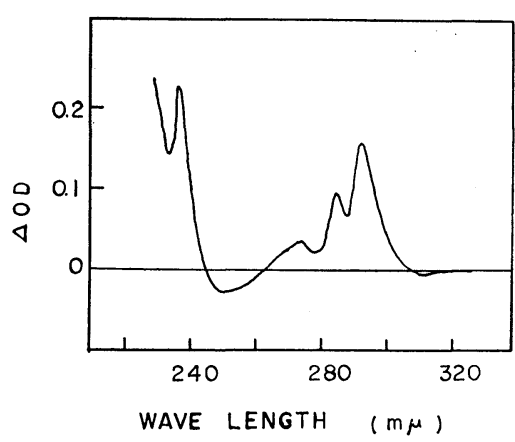

Fig. 1. Difference spectrum of solvent perturbation by $43 \%$ glucose at $\mathrm{pH}$ 6.0. The concentration of PI was $3.95 \times 10^{-4} \mathrm{M}\left(\mathrm{OD}_{280}=2.3\right)$.

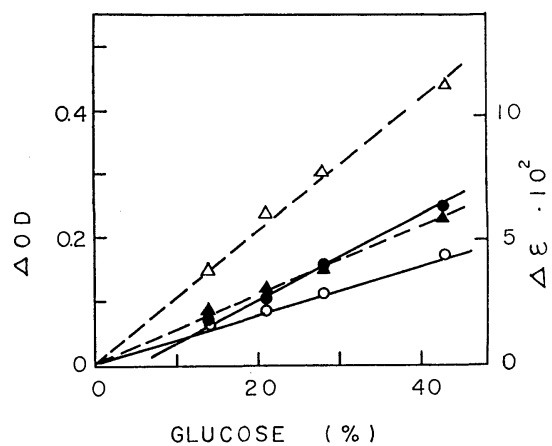

Fig. 2. Solvent perturbation of PI by glucose at $\mathrm{pH}$ 6.0. The concentration of tryptophan and PI was 3.95 $\times 10^{-4} \mathrm{M}$, respectively.

○: $\Delta \mathrm{OD}_{292}$ of $\mathrm{PI}$.

$\triangle: \Delta \mathrm{OD}_{291}$ of tryptophan.

๑: $\Delta \mathrm{OD}_{235}$ of $\mathrm{PI}$.

$\Delta$ : $\Delta \mathrm{OD}_{230}$ of tryptophan.

*) The concentration of the polymer was conveniently represented on the basis of moles of tryptophan residue.

**) For abbreviation of copolyamino acid (see [8]).

***) $\Delta$ OD: intensity of difference spectrum in absorbancy units, $\Delta \varepsilon:$ molar extinction coefficient of difference spectrum. 


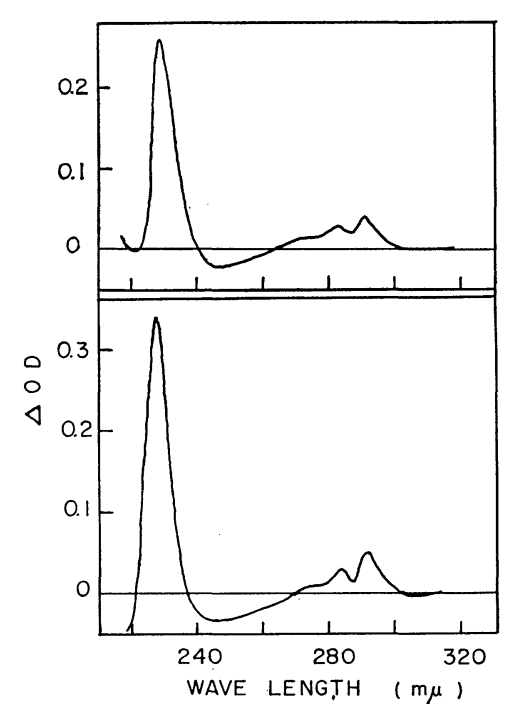

Fig. 3. Difference spectra of solvent perturbation by $43 \%$ glucose at $\mathrm{pH}$ 6.0. The concentration of tryptophan and PI was $1.13 \times$ $10^{-4} \mathrm{M}$, respectively.

Top: PI, bottom: tryptophan.

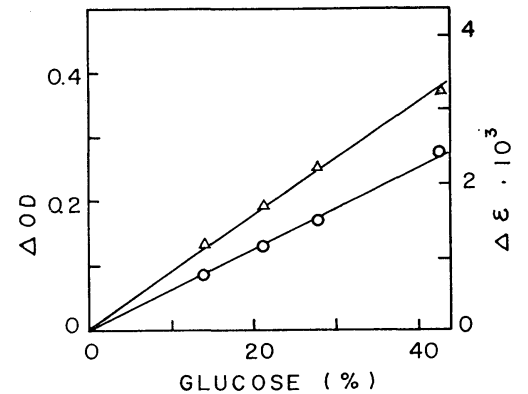

Fig. 4. Solvent perturbation of PI by glucose at $\mathrm{pH}$ 6.0. The concentration of tryptophan and PI was $1.13 \times 10^{-4} \mathrm{M}$, respectively. O: $\Delta \mathrm{OD}_{228}$ of $\mathrm{PI}$.

$\triangle: \Delta \mathrm{OD}_{228}$ of tryptophan. to the machine capacity. Typical difference spectra of tryptophan and $\mathrm{PI}$ at $1.13 \times 10^{-4} \mathrm{MI}$ are shown in Fig. 3 . If the $\triangle \mathrm{OD}$ measured around $230 \mathrm{~m} \mu$ truly represents that of the difference spectrum, it must be a linear function of the concentration of solute to be perturbed. In fact, $\Delta \mathrm{OD}$ around $230 \mathrm{~m} \mu$ measured at concentration of $1.13 \times 10^{-4} \mathrm{M}$ fitted on a line in plot of $\triangle \mathrm{OD} v \mathrm{~s}$ the concentration of solute. In Fig. 4, $\Delta \mathrm{OD}_{228}$ 's are plotted as a function of the glucose concentration at $\mathrm{pH} 6.0$. The degree of solvent-accessibility of tryptophan residues was calculated to be 69 and $70 \%$ from Figs. 2 and 4, respectively. Thus, when the concentrations of polymer and tryptophan are suitably chosen, $\Delta O D$ near $230 \mathrm{~m} \mu$ and $\Delta \mathrm{OD}$ near $290 \mathrm{~m} \mu$ give the same value to the solvent-accessibility of tryptophan residues. The same experiment was carried out at $\mathrm{pH} 8.0$ and the degree of solventaccessibility was calculated to be $66 \%$. At pH 5.0, all tryptophan residues came into contact with solvent. The $\mathrm{pH}$-dependence of the degree of solvent-accessibility of tryptophan residues of $\mathrm{PI}$ is shown in Fig. 5.

Solvent perturbation of PII.*) The solvent-accessibility of tryptophan residue of PII measured in glucose solution was nearly the same as those of PI at pHs 6.0 and 8.0. At pH 5.0, PII showed $70 \%$ of solvent-accessibility. The solvent perturbation of the absorption spectrum of protein is affected by the molecular size of perturbant. Accordingly, sucrose was used to elucidate the cause of anomaly of PII at $\mathrm{pH}$ 5.0. No discrepancy, however, was observed between glucose

*) Poly $\operatorname{Trp}^{5} \mathrm{Ala}^{24} \mathrm{Glu}^{72}$. 
and sucrose solutions. The results for PII are also shown in Fig. 5.

Charge effect on $U V$-spectrum of PII. The difference spectrum due to the charge effect was not observed. This confirms the fact that the solvent-accessibility of tryptophan residues of PII was independent of $\mathrm{pH}$ value of medium.

Solvent perturbation of lysozyme by glucose. The solvent-accessibility of tryptophan residues of lysozyme was found to be $55 \%$ from $\Delta \mathrm{OD}_{293}$ and $29 \%$ from $\Delta \mathrm{OD}_{232}$. The solvent-accessibility of the tryptophan residues of lysozyme had been found to be about $60 \% .^{5)}$ Thus, it is clear, against expectation, that the $\Delta \mathrm{OD}_{232}$ cannot be simply used for the estimation of

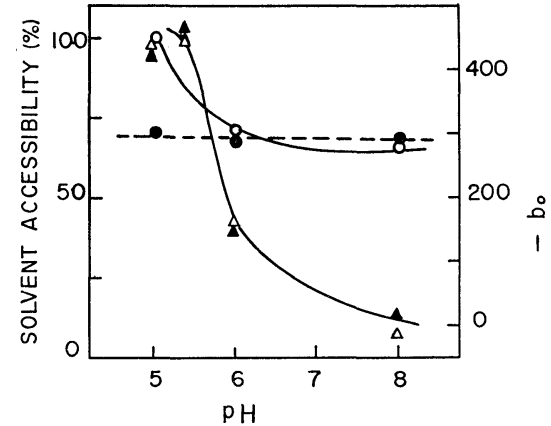

Fig. 5. pH-Dependence of degree of solvent-accessibility of tryptophan residues of the polymers.

$O, \triangle:$ degree of accessibility and $\mathrm{b}_{0}$ of PI.

- $\mathbf{\Delta}$ : degree of accessibility and $b_{0}$ of PII. solvent-accessibility of tryptophan residues of protein.

Optical rotatory dispersion. Moffitt's parameter, $\mathrm{b}_{0}$, of PI and PII at $\mathrm{pH} 5.0$ was -450 and -420 , respectively.

Discussion. A polyamino acid composed mainly of glutamyl residue has $\alpha$-helix structure at $\mathrm{pH}$ 5.0, where nearly all $\gamma$-carboxyl groups are protonated. If there is no aggregation of helix columns, all tryptophan residues may come into contact with the medium. This was true for PI. In the high $\mathrm{pH}$ region, where all the carboxyl groups are ionized, ${ }^{7)}$ the polyamino acid containing the bulk of side chain carboxyl groups was known to take a random structure because of the electrostatic repulsion between carboxylates. The fact that about $30 \%$ of the tryptophan residues of PI and PII was masked from contact with the medium in neutral region implies the presence of some kind of regular structure. Thus, freezing of the hydrophobic side chain such as indole nucleus of tryptophan residues may correlate with the formation of the polyfunctional site in a polyamino acid. The degree of solvent-accessibility of the tryptophan residues of PII was independent of the $\mathrm{pH}$ value of medium. This was confirmed by the results of solvent perturbation by sucrose and the charge effect. The masking of $30 \%$ of the tryptophan residue of PII at $\mathrm{pH} 5.0$ was assumed to be accompanied by the association of helix columns, because $b_{0}$ value indicates that PII has helix structure at $\mathrm{pH} 5.0$.

Optical density of a peak around $230 \mathrm{~m} \mu$ in the difference spectrum of the polymer or amino acid tryptophan was completely proportional 
to that around $290 \mathrm{~m} \mu$. It was concluded that the exposure of buried tryptophan residues in the polymer molecule causes the appearance of the peak around $230 \mathrm{~m} \mu$. Thus, the optical density of the peak around $230 \mathrm{~m} \mu$ in the difference spectrum cannot be a direct index of the regular structure of polypeptide chain. Furthermore, it was shown that the optical density of the peak around $230 \mathrm{~m} \mu$ in difference spectrum cannot simply be used for defining the state of the tryptophan residues in a protein molecule, because many chromophores contribute to the absorbancy around $230 \mathrm{~m} \mu$.

\section{References}

1) Fasman, G. D.: "Poly- $\alpha$-amino Acid" Maecel Dekker Inc. N. Y. (1967).

2) Glazer, A. N., and Smith, E. L.: J. Biol. Chem., 235, PC43 (1960).

3) Herman, J., and Scheraga, H. A.: J. Am. Chem. Soc., 82, 5156 (1960).

4) Bigelow, C. C., and Geschwind, I. I: Compt. rend. trav. lab. Carlsberg, 31, 2831 (1960).

5) Hamaguchi, K., Hayashi, K., Imoto, T., and Funatsu, M.: J. Biochem., 55, 24 (1964).

6) Moffitt, W., and Yang, J. T.: Proc. Natl. Acad. Sci. U. S., 42, 296 (1956).

7) Doty, P., Wada, A., and Yang, J. T.: J. Polymer Sci., 23, 651 (1957).

8) IUPEC-IUB: Biochemistry, 7, 483 (1968). 